\title{
MULTIVARIABLE FUZZY FEEDFORWARD COMPENSATION
}

\author{
R. Ferreiro García, F. J. Pérez Castelo, J. Vidal Paz \\ Dep. Industrial Engineering. University of A Coruna \\ E.S Marina Civil, Paseo de Ronda 51. 15011- A Coruna. Spain \\ fax: 3481167101 \\ e-mail: ferreiro@udc.es \\ ${ }^{\dagger}$ ECC2001 Conference \\ e-mail: ecc2001efe.up.pt \\ http://www. Ie.up.pt/ecc2001/
}

Keywords: Non-linear feedforward, multivariable disturbances, multivariable feedforward compensation, fuzzy feedforward compensation

\begin{abstract}
This work deals with some practical aspects of fuzzy PID controllers regarding multivariable non-linear feedforward compensation. This contribution concerns to the task of compensation for multivariable disturbances on the controlled variable. The strategy consist in establishing a fuzzy rule base for all disturbance variables and compensate the control variable by feedforward action by means of a fuzzy rule base properly adjusted.
\end{abstract}

\section{Feedforward control background}

Conventional feedforward control deals with the task of correct the manipulated variable for a disturbance on the controlled process. Most common industrial processes are disturbed by more than a variable. For instance, in heat exchangers, controlled temperature is disturbed from flow and temperature variations of heated fluid. Furthermore, they are disturbed also by variations of the operating point because dissipation heat may depend on the ambient and operating temperatures and by process parameters.

In conventional feedforward control [2,3], an error must be detected in a controlled variable before the feedback controller can act to change the manipulated variable. Therefore, disturbances must upset the system before the feedback controller can do anything. It seems very reasonable that if a disturbance entering a process could be detected, a controller should begin to correct for it before it upsets the process. This is the basic idea of feedforward control. If disturbance can be measured, this result will be used to send a signal through a feedforward control algorithm that makes appropriate changes in the manipulated variable so as to keep the controlled variable near its desired value.
Classical industrial controllers offer the possibility of compensation for only a disturbance variable entering the process, if such a disturbance can be measured. The real problem concerning industrial control, in which a good performance is needed, requires the compensation task for more than a single disturbance variable. In such case conventional controllers are not efficient and fuzzy logic controllers takes advantage. Furthermore, disturbance variables are associated by non-linear functions. Non-linear feedforward compensator can be designed for non-linear systems.

An alternative to implement feedforward control systems in order to compensate multivariable disturbances, may be implemented by means of a rule based controller, for which the use of fuzzy logic is a powerful tool.

\section{Rule based feedforward compensation}

In complex systems, analytical methods to find non-linear feedforward controllers become too complex. Alternative solutions based in numerical techniques must be used to find the required non-linear changes in manipulated variables. One of numerical techniques consists in apply rule based compensators. In Figure 1 it is shown the general structure if a multivariable feedforward compensator.

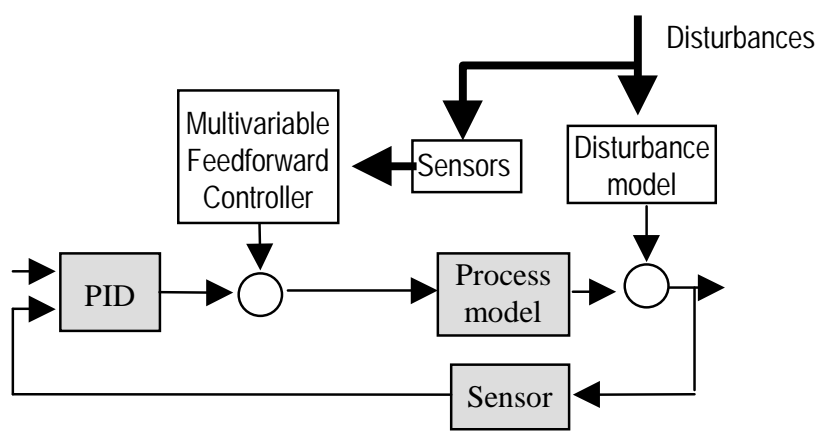

Figure 1: Feedforward feedback control structure. 
In Figure 2 it is shown the case of a system disturbed by two different variables $n 1$ and $n 2$, which enter the fuzzy rule base responsible for generate a corrective output to be added to the output of the feedback controller.

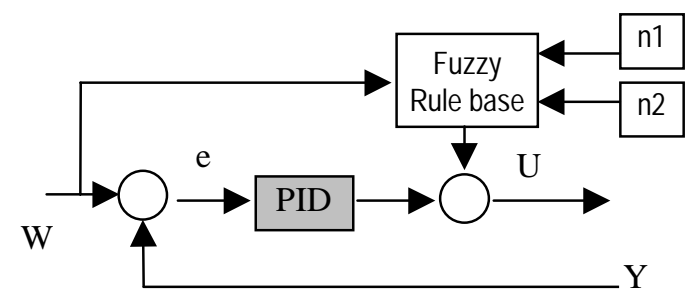

Figure 2: Multivariable feedforward compensation using a fuzzy rule base.

Given a closed loop system disturbed by a function consisting in a set of non-linear variables, its open loop model is defined as

$$
\frac{Y(s)}{U(s)+\sum n(s)}=G(s)
$$

where $Y(s)$ is the system output or response, $U(s)$ is the total controller output, $\Sigma n(s)$ are the disturbances and $G(s)$ is the system transfer function. (1) can be expressed as

$$
Y(s)=\left(U(s)+\sum n(s)\right) G(s)
$$

The condition to ensure the steady state response due to the association of two exciting variables, which are the output of a feedback controller and the set of disturbance variables $(U+$ $\Sigma n)$ is that

$$
(U+\Sigma n)=\text { constant }
$$

which implies that

$$
\Delta U+\Delta \Sigma n=\text { constant }
$$

or

$$
\Delta \Sigma n=-\Delta U
$$

Expression (5) means that any variation of disturbance variables $\Delta \Sigma n$, must be compensated by $-\Delta U$ in order to keep the controlled system in steady state. $-\Delta U$ will be generated by a fuzzy rule base.

Expression (5) will be implemented by means of a fuzzy rule base [1].

\section{An application}

According Figure 3, for a heat exchanger, the first law can be written as

$$
Q=M . C e \frac{d T}{d t}+q_{F} . C e\left(T-T_{i}\right)+K_{T}\left(T-T_{e}\right)
$$

rearranging (6) to separate disturbances

$$
Q+q_{F} \cdot C e \cdot T_{i}+K_{T} T_{e}=M \cdot C e \frac{d T}{d t}+\left(q_{F} \cdot C e+K_{T}\right) T
$$

where $M$ is the mass of fluid into the heat exchanger, $C e$ is the specific heat of fluid, $K_{T}$ is the total transfer coefficient between exchanger and environment, $q_{F}$ is the fluid flow, $T i$ is the input fluid temperature, $T e$ is the environment temperature and $\mathrm{T}$ is the output fluid temperature.

In this study it is considered that only variations exist in fluid temperature and flow, so that environment temperature remains constant.

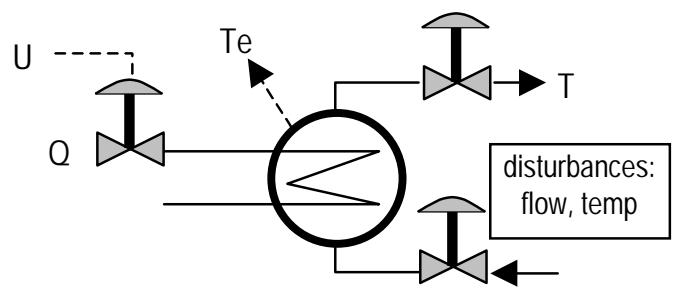

Figure 3: Heat exchanger affected by disturbance variables (fluid flow and input fluid temperature).

The block diagram for system simulation is shown in Figure 4. According this figure, the feedback controller output is added to the output of the fuzzy rule base responsible for compensate the variations in the fluid flow and fluid temperature. As it is shown in the left hand side of expression (7), fluid flow and fluid temperature are associated by means of a product, which result in non-linear function for mentioned disturbance variables

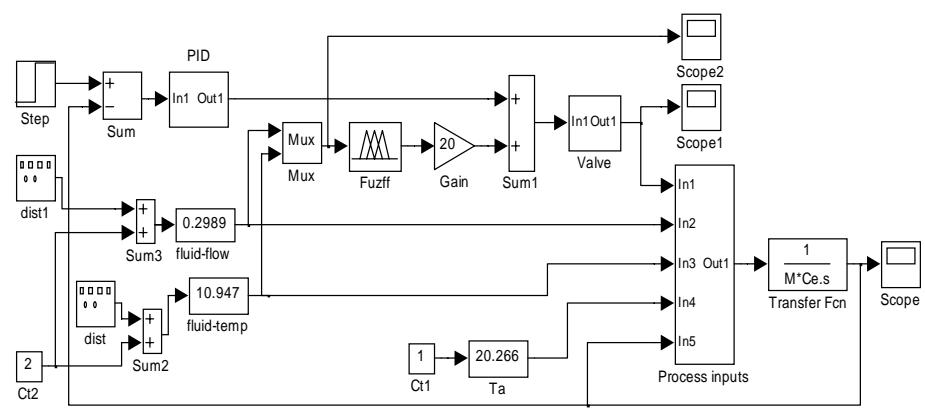

Figure 4: Block diagram for system simulation.

The rule base is quite simple, provided that only nine rules are sufficient to compensate the disturbances studded. The Table 1 shows the full rule base.

The 3D rule base input/output relationship is shown in Figure 5 .

Rule base adjusting task has been carried out off-line by simulation. For several discrete values of the steady state variables related with feedforward compensation (flow, input temperature and rule base output) stored into a data file, a 
neuro fuzzy toolbox, the NeuroSys of Siemens is applied for such training procedure.

\begin{tabular}{|l|}
\hline If (flow is low) and (temp is low) then (output is Z) \\
\hline If (flow is low) and (temp is med) then (output is Vlow) \\
\hline If (flow is low) and (temp is high) then (output is Low) \\
\hline If (flow is med) and (temp is low) then (output is Vlow) \\
\hline If (flow is med) and (temp is med) then (output is Med) \\
\hline If (flow is med) and (temp is high) then (output is high) \\
\hline If (flow is high) and (temp is low) then (output is Med) \\
\hline If (flow is high) and (temp is med) then (output is high) \\
\hline If (flow is high) and (temp is high) then (output is Vhigh) \\
\hline
\end{tabular}

Table1: Rule base

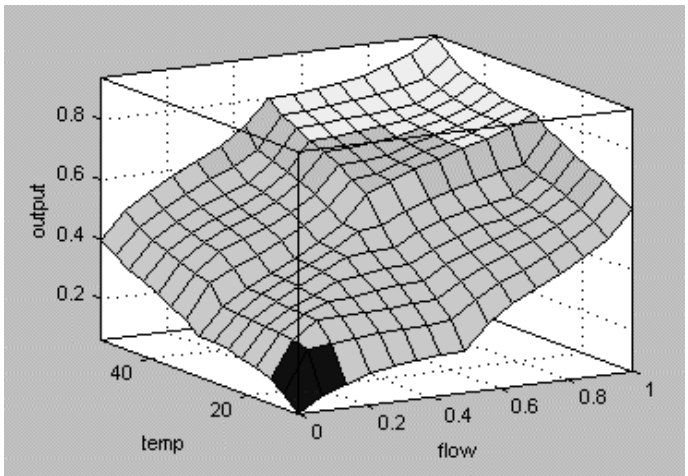

Figure 5: Block diagram for system simulation.
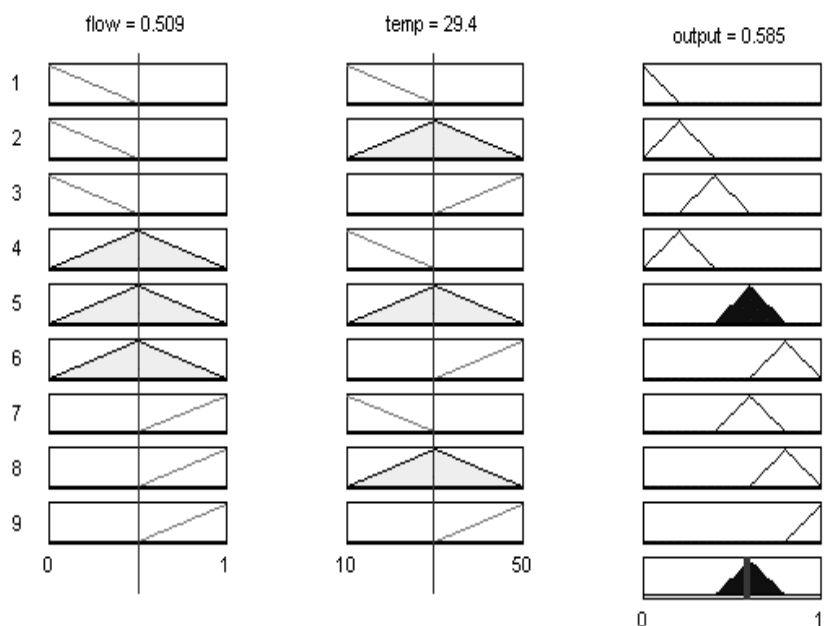

Figure 6: The rule base layout.

\section{Simulation results}

Simulation is performed by setting the input set point in a specified value, while disturbance variables (fluid flow and fluid temperature are varying according the curves shown in Figure 8. Fluid flow variations consist in a sine function of .2 $\mathrm{Kg} / \mathrm{s}$ amplitude and $0.002 \mathrm{rad} / \mathrm{s}$. frequency. Fluid temperature consist in a sine function of 4.5 degrees amplitude and frequency $0.004 \mathrm{rad} / \mathrm{s}$.

Results are shown in Figure 7. They show that disturbances are compensated by means of the fuzzy controller with satisfactory response values.

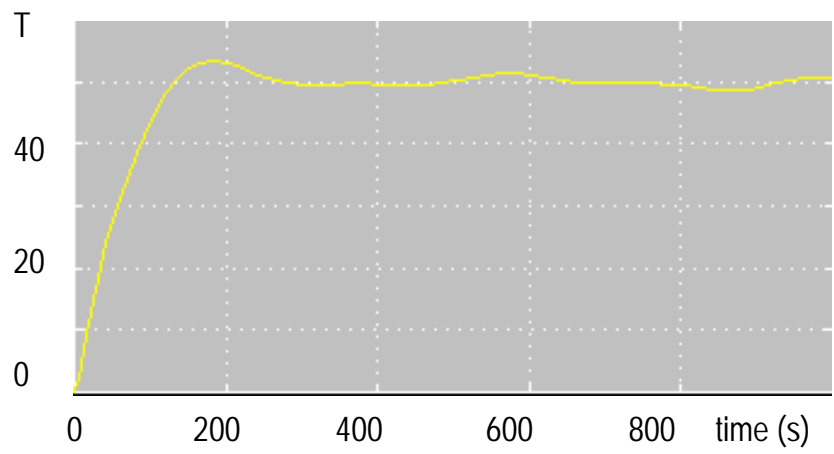

Figure 7: Time response to a step set point input with disturbances in fluid flow and fluid temperature $\mathrm{q}_{\mathrm{F}}, \mathrm{T}_{\mathrm{i}}$

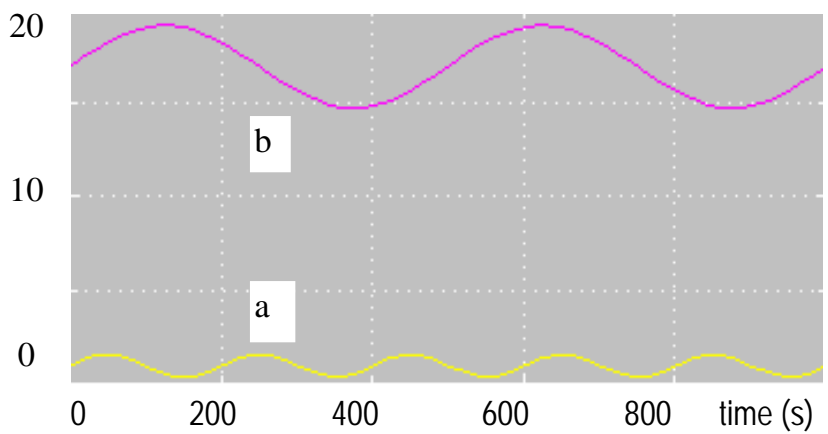

Figure 8: Variation of disturbance variables.

(a) flow, (b) temp

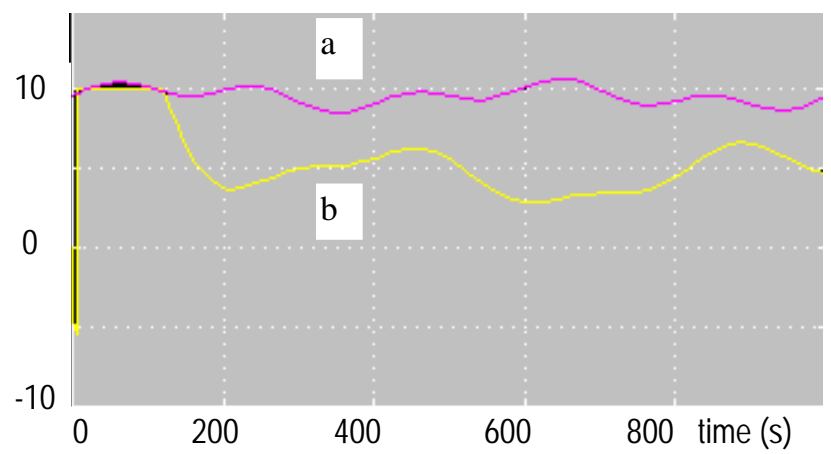

Figure 9: Control variables dynamics:

(a) PID output. (b) Fuzy rule base output 


\section{Conclusions}

Multivariable non-linear feedforward compensation by means of a fuzzy rule base can be succefully achieved. To get the fuzzy rule base properly adjusted it is necessary to select between two options: For the case of a dterministic mathmodel of disturbed process, simulation can be used to find the input/output relationship. In other cases neuro-fuzzy adjusting procedures must be performed. In any case disturbances mus be accessible to measurement.

\section{References}

[1] King R.E. "Computational Intellingence in Control Engineering”, Chap.8, pp.105-118, Marcel Decker, Inc. New York, U.S.A. (1999).

[2] Luyben W.L. "Process modeling, simulation, and control for chemical engineers", Chap.11, pp.383-391, McGraw-Hill, Inc U.S.A. (1989).

[3] Shinskey F.G.. "Process control systems. Applications, design and tuning”, Chap.7, pp.267-309, McGraw-Hill, Inc U.S.A. (1996). 